\title{
Sidelink Technologies Comparison for Highway High-Density Platoon Emergency Braking
}

\author{
Guillaume Jornod ${ }^{* \dagger}$, Tianxiang $\mathrm{Nan}^{\dagger}$, Michael Schweins ${ }^{\dagger}$, \\ Ahmad El Assaad*, Andreas Kwoczek* and Thomas Kürner ${ }^{\dagger}$ \\ ${ }^{*}$ Group Research, Volkswagen AG, Wolfsburg, Germany \\ \{guillaume.jornod,ahmad.el.assaad,andreas.kwoczek\}@volkswagen.de \\ $\dagger$ Institut für Nachrichtentechnik, Technische Universität Braunschweig, Braunschweig, Germany \\ \{nan,schweins,kuerner\}@ifn.ing.tu-bs.de
}

\begin{abstract}
We present a benchmarking framework for different radio access technologies (RATs) in a high density platooning (HDPL) emergency braking use case. We assess the performance of IEEE 802.11p as well as LTE-V managed mode (mode 3) and unmanaged mode (mode 4) for sidelink communications. The performances are studied in terms of delays, packet error rates (PERs) and functional safety indicators. We first vary the number of vehicles, the surrounding traffic and the inter-vehicle distance. Multiple traffic scenarios are then investigated for the most challenging conditions. We find that for reasonable surrounding traffic, the platoon is generally safe in this emergency scenario, although packet error rates are growing for IEEE 802.11p and LTE-V mode 4 as the traffic intensifies, along with delays for the former technology. Thanks to scheduling, LTE-V mode 3 is not affected by this increasing PER and shows a large constant delay: the scheduling delay. With this study, we pave the way for a further study of these radio technologies with more accurate channel models as well as including new $5 \mathrm{G}$ components in our benchmarking.
\end{abstract}

\section{INTRODUCTION}

Vehicle-to-everything (V2X) communication is one of the key features of Intelligent Transportation Systems (ITS) as it provides wireless connectivity for road users, such as vehicles, their passengers, infrastructure and pedestrians. The 3GPP consortium made available the release 15 for the fifth generation of cellular communication technologies (5G). The V2X 5G features are expected to be part of the upcoming releases 16 and 17 . To prepare the assessment of this upcoming technology, we propose a benchmarking setup for existing direct communications radio technologies such as IEEE $802.11 \mathrm{p}$, LTE-V with and without the assistance of the network.

These technologies are here challenged in the context of a High-Density Platooning (HDPL) system performing an emergency braking on a highway. This use case is analyzed under different platooning settings such as the number of trucks, their inter-vehicle distances (IVDs) and various surrounding traffic densities. We assess the performance of the three aforementioned technologies in terms of three key

This work has been supported in part by the Federal Ministry of Education and Research of the Federal Republic of Germany (BMBF) in the framework of the project 5G NetMobil with funding number 16KIS0681. The authors alone are responsible for the content of the paper. performance indicators (KPIs): (i) delays, (ii) packet error rates (PERs) and (iii) IVDs during the emergency maneuver.

The key contributions of this report are:

1) Proposing a benchmarking framework for radio access technologies (RATs);

2) Analyzing the influence of the environment on the performance of these technologies;

3) Showing the advantages and drawbacks of each technology in the scope of our use case.

The remaining of this paper is organized as follows: in Sec. II, the simulation platform is introduced and the RATs presented; Sec. III develops the HDPL emergency braking use case in further details; the simulated scenarios are presented and their results described in Sec. IV; in Sec. V, these results and the assessment of the RATs are discussed; finally, Sec. VI summarizes the benchmarking framework and the key findings.

\section{Simulation Platform}

In this section we first introduce our simulation platform, an integrated system of network simulator and traffic simulator considering the real-time vehicle mobility and communication. We then present the RATs used in this benchmarking framework.

\section{A. Integrated Simulation System}

Before introducing our simulator choices and their interactions, we provide a rapid survey of existing tools.

1) State of the Art: The combination of coupled network and vehicle simulators is a topic under active development in the research community.

For instance, the Veins framework [1] provides the coupling of the network simulator OMNeT++ [2] and the urban traffic simulator Simulation of Urban Mobility (SUMO) [3] (which will be further discussed). Originally only proposing the implementation of IEEE $802.11 \mathrm{p}$, it has been extended to also provide LTE [4] as well as ITS-G5 facilities [5] simultaneously. A platooning extension of this framework is proposed by PLEXE [6]. However, the LTE implementation, SimuLTE [7], does not provide standard-conformant device-to-device (D2D) implementing unmanaged direct communications [8]. 


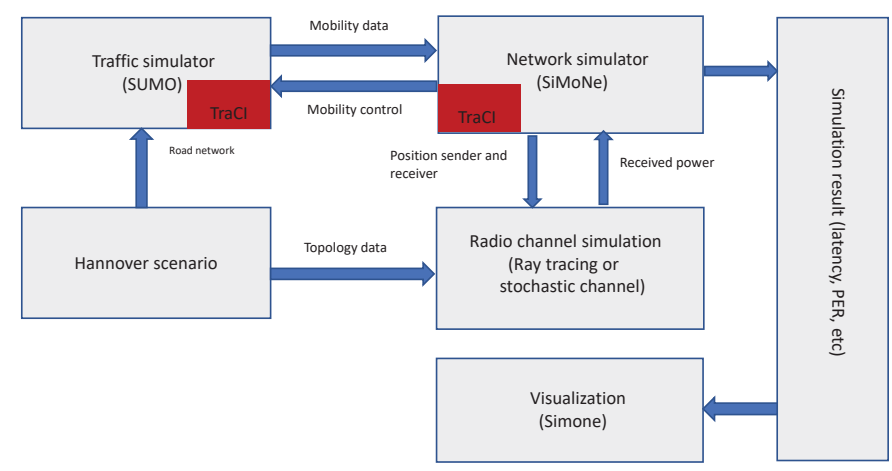

Fig. 1. Structure of integrated simulation platform.

An alternative to OMNet++ is ns-3 [9]. Frameworks coupling ns-3 with SUMO include OVNIS [10] and the results of the EC funded project iTETRIS [9]. Whilst these platforms provide precise implementations of communications technologies, with for instance a standard-conformant LTE direct link implementation [11], the interfaces are not under active development. Combinations with sub-microscopic vehicles simulators, such as with Webots [12], also exists, but are more focused on the development of autonomous vehicle algorithms than on the communications technologies themselves [13].

2) Traffic Simulator: SUMO [3] is chosen as the traffic simulator for its ability to generate and simulate road networks and traffic. These simulation capabilities include car following, lane changes, overtaking of cars, acceleration and deceleration.

SUMO is a microscopic vehicle traffic simulator. Contrary to macroscopic models which consider probabilistic vehicles model under constraints such as roads, streets, crossroads, and traffic lights, microscopic approaches, instead, focus on the movement of each individual vehicle. They do not, however, consider the internal functioning of the vehicles, as submicroscopic simulators such as Webots [12] do. In SUMO, the target speed and subsequent position of each vehicle can be calculated based on the status of other vehicles as well as the street properties such as speed limitations. The simulations in SUMO are time-discrete, i.e. based on a specific time resolution, and space-continuous.

3) Network Simulator: Network simulators are able to evaluate the performance of network protocols and the communicated traffic under dynamic radio channel conditions. We use the Simulator for Mobile Networks (SiMoNe), a network simulator developed by the Institute for Communications Technology at TU Braunschweig. The platform was originally developed as an administrative platform for simulation scenarios and to access large amounts of geodata. It was gradually expanded with simulative abilities. SiMoNe holds all the data in a database, e.g. the transmission technologies, the frequency bands, the cell positions and the mobility traces [14].
TABLE I

IEEE 802.11P KEY PARAMETERS

\begin{tabular}{l|c} 
Parameter & Setting \\
\hline \hline Transmission power & $23 \mathrm{dBm}$ \\
\hline Congestion Window (CW) & {$[0,15]$} \\
\hline Time slot & $13 \mu \mathrm{s}$ \\
\hline Short Inter-frame Space (SIFS) & $32 \mu \mathrm{s}$ \\
\hline Arbitration Inter-frame Space Number (AIFSN) & {$[2,7]$} \\
\hline Bandwidth allocation & G5-CCH
\end{tabular}

SiMoNe extends its database feature by interfacing with other simulators. Over the aforementioned network simulators, SiMoNe has the advantage of proposing IEEE 802.11p and LTE-V (mode 3 and 4) as well as implementing both stochastic, such as WINNER + [15], and ray tracing channel models. Using the Traffic Control Interface (TraCI), SiMoNe can process the vehicular traffic data of SUMO. Although SUMO implements some vehicle following algorithms, we also use TraCI to control a specific set of vehicles using a custom control algorithm. The advantage of such a setup is to be able to consider the communicated information in the control system and to directly compute the KPIs in the network simulator. The structure of our simulation platform is shown in Fig. 1.

\section{B. Communication Models}

The communications between vehicles is performed using three RATs, viz. IEEE 802.11p, LTE-V mode 3 and LTE-V mode 4.

1) IEEE 802.11p: Messages between vehicles are sent with the IEEE 802.11p technology and the Carrier Sense Multiple Access with Collision Avoidance (CSMA/CA) channel access method [16]. The key parameters used in the simulation are presented in Tab. I. The frequency allocation in our simulation is based on the allocation in the European Union [17]. In this allocation standard, the usage of G5-CCH and G5-SCH1 to G5-SCH2 are dedicated to ITS road safety. For our purpose, we only consider the G5-CCH band.

The basic principle in CSMA/CA is called listen before talk (LBT). A vehicle that plans to transmit listens to the channel and assesses its utilization. The transmitting device compares the received energy with a defined threshold value. If the channel is detected to be free, the device immediately starts the transmission. Else, the device waits until the channel is free again. At his point, in CSMA, a receiver does not start its transmission immediately. It executes the so-called Backoff process: A random number is first drawn and used to define an idling time after the channel is free again. This prevents message collision from multiple waiting vehicles performing immediate transmission. With this idle time, the probability that only one vehicle starts the transmission is increased, thus reducing the probability of collision.

Messages are broadcast to all possible receivers by default. Platoon unicast messages are discarded by non platoon members. For all messages the transmission speed was configured to $6 \mathrm{Mbit} / \mathrm{s}$. Channel coding and decoding is not modelled 
for complexity reasons. Instead, a threshold for the signalto-interference-plus-noise ratio (SINR) is defined. An error free reception is assumed if this threshold is exceeded (see Sec. II-C). Only the successful reception of unicast messages in the platoon are answered with an acknowledgment (ACK) to inform the transmitter about the reception. In case of a message collision, a timer overflow at the receiver indicates the missing ACK and will initiate a retransmission of the unicast packet.

2) LTE-V: For LTE-V, two modes are implemented, mode 3 (managed mode) and mode 4 (unmanaged mode) [18]. In the former mode, resource scheduling and interference management on the direct communication links are assisted by Evolved Node B (eNB) over the Uu interface. In the latter mode, this is done by semi-persistent scheduling. Both modes use the PC5 interface for the communication using a frequency of $3.4 \mathrm{GHz}$ and a signal bandwidth of $10 \mathrm{MHz}$. With this bandwidth, 96 resource blocks are available per subframe, four being held by overheads. While applications may generate messages at any given time, the transmission with LTE is bounded to the resource grid with a time base of $1 \mathrm{~ms}$.

a) Mode 3: Each cell holds a list of scheduling requests (SR) for its connected subscribers. All SRs in the list are first sorted by priority, viz. by maximum allowed message delay. The eNB then assigns - in a random manneravailable resources to messages and creates corresponding sidelink control information (SCI). Assigned SRs are then deleted from the list. Remaining SRs stay in the list and will be assigned at the next scheduling step. eNBs are assumed to introduce a fixed delay of $12 \mathrm{~ms}$ for traffic requests signalling and processing time.

b) Mode 4: The resource management in LTE-V mode 4 makes use of Sensing-Based Semi-Persistent Scheduling (SBSPS) [19], [20]. Within a time span of 100 subframes, subscribers reserve resources for a number of consecutive reselection counter packet transmissions, which are randomly initialized from $[5,15]$. Subscribers transmits SCIs and counter values along with their messages within their reserved resources. Sharing SCIs allow the evaluation of available resources when making reservations. Subscribers are forced to schedule their EMs (see Sec. III-A and Tab. II) within the range of maximum delay, here $20 \mathrm{~ms}$. A new resource reservation is required when the reselection counter reaches zero or when the timespan of the current reservations is greater than the maximum message delay. Note that prioritized messages such as platoon emergency messages and commands are sent twice within an interval of $\pm 15 \mathrm{~ms}$ around the reserved resources.

\section{Transmission Evaluation}

We use the WINNER+ model [15] for both IEEE 802.11p and LTE-V. This model covers the channel model with several propagation scenarios including multipath components from stochastic clusters. Our technology implementations are standard-conformant down to the MAC layer. Indeed, we omit coding / decoding and use an SINR threshold mechanism to determine whether a packet is transmitted. The throughput of a resource block is limited by the receiver sensitivity and therefore by a SINR threshold which indicates whether a message can be decoded. In IEEE 802.11p (resp. LTE-V), with a transmission power of $23 \mathrm{dBm}$, this SINR threshold is set to $6 \mathrm{~dB}$ (resp. $10 \mathrm{~dB}$ ). These values are crucial parameters as they determine the potential receivers for which the transmission will be evaluated, and for which the KPI will be computed. We base our threshold choices on the work presented in [21] and [22] for IEEE 802.11p and LTE respectively. Interference can be caused by subscribers who simultaneously occupy the same time and frequency resources. Furthermore, subscribers are not able to simultaneously send and receive.

\section{USE CASE}

We evaluate the performance of the RATs in the scope of a HDPL emergency braking scenario. First, the platooning algorithm and the emergency braking are described. Then, the traffic environment in which this situation takes place is presented.

\section{A. Platoon Emergency Braking}

The platoon operation is based on two levels of control, one global, operated by the so-called platoon leader (PLL), and one local, operated at each platoon member (PLM). The relationship between the two control scopes is supported by the exchange of messages, which is described in the following. PLL chooses an IVD, which is transmitted within a Leader Command (LC). Each PLM is then responsible to keep its forward IVD, thanks to sensor and communicated information. PLMs send periodic messages (PMs) containing pose and dynamics information. In our scenario, PLL is not necessary the first PLM.

At a specific timestep, an emergency is detected by the first PLM, which triggers its local emergency braking while also broadcasting an emergency message (EM). Since we investigate the impact of communication delay, we assume that the global braking maneuver is coordinated by PLL and is triggered by the EM reception. PLL then coordinates the global maneuver by sending commands through LCs. In this use case, the reception of the LC is crucial as the farther from the first PLM, the larger the reaction time if no communication is implemented. Tab. II summarizes the platoon messages and their transmission modes.

In SiMoNe, the highest time resolution for message transmission and command execution is $10 \mathrm{~ms}$. To mimic realworld hardware and software delays, some states transitions are delayed by values drawn from uniform distributions.

\section{B. Traffic Scenario}

The platoon is driving on the German Highway A2, in the Hannover scenario presented in [14]. Using the conversion tool embedded in SUMO, the road network is generated from Open Street Map (OSM) data. The area of interest is delimited in red in Fig. 2, around which we place some LTE eNBs. The considered highway section has a length of $3650 \mathrm{~m}$ and has 
TABLE II

Platoon MESSAGES

\begin{tabular}{l|l|l|c|c} 
Message & Acronym & Description & Transmission mode & Size \\
\hline \hline Cooperative Awareness Message & CAM & From ITS-G5 standard & Broadcast & $400 \mathrm{~B}$ \\
Periodic Message & PM & Pose and dynamics info. & Unicast & $400 \mathrm{~B}$ \\
Emergency Message & EM & Sent by first platoon vehicle to indicate hazard & Broadcast & $150 \mathrm{~B}$ \\
Leader Command & LC & Control message from PLL & Unicast & $150 \mathrm{~B}$
\end{tabular}

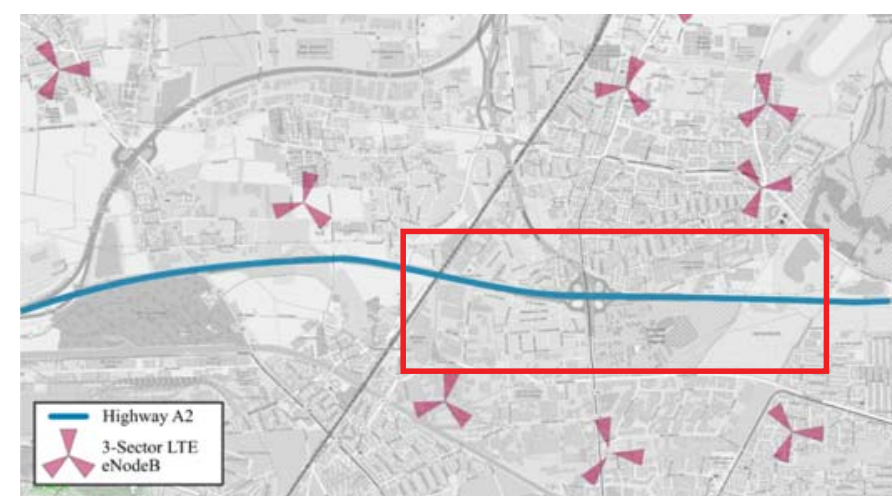

Fig. 2. Positions of eNBs in SiMoNe. The red rectangle delimits the scenario area.

three lanes in each direction. As SUMO requires a value for the maximal speed on the highway, the unlimited speed limit cannot be modeled and the value is set to $120 \mathrm{~km} / \mathrm{h}$.

To model the surrounding traffic, we add vehicles on the road network. These vehicles are distributed on the highway and on the other roads close to it in order to simulate a realistic channel and system load. Tab. III summarizes the SUMO parameters used to model these vehicles. Note that the control algorithm and maximum accelerations of the platoon members are overridden by the control from SiMoNe through TraCI.

To challenge the communication systems, we vary the density of the surrounding traffic. Indeed, this density may have a large impact on the channel load and on the links quality. Using data from traffic volume maps in Germany, we define the following: (i) low volume with $V=36,000$ vehicles $/ 24 \mathrm{~h}$ and (ii) medium volume with $V=72,000$ vehicles $/ 24 \mathrm{~h}$. The density of the surrounding traffic is then obtain as $\delta=V / 24 \nu L$, where $\nu$ is the mean speed and $L$ the number of lanes. With $\nu=120 \mathrm{~km} / \mathrm{h}$ and $L=3$, this leads to (i) $\delta=4$ vehicles $/ \mathrm{km} /$ lane for the low density and (ii) $\delta=8$ vehicles $/ \mathrm{km} /$ lane for the medium one. Each vehicle from this surrounding traffic transmits CAMs periodically. The sending frequency is proportional to the vehicle speed, between 1 and $10 \mathrm{~Hz}$. This upper boundary is reached by most of the vehicles.

\section{Simulations And Results}

In this section we describe the specific scenarios we simulate and with which KPIs we assess their performance before presenting the results.
TABLE III

VEHICLE PARAMETRIZATION IN SUMO

\begin{tabular}{l||c|c} 
Type & truck & passenger car \\
\hline Length & $16.5 \mathrm{~m}$ & $4.3 \mathrm{~m}$ \\
\hline Width & $2.55 \mathrm{~m}$ & $1.5 \mathrm{~m}$ \\
\hline Max. speed & $80 \mathrm{~km} / \mathrm{h}$ & $160 \mathrm{~km} / \mathrm{h}$ \\
\hline Target velocity & $72 \mathrm{~km} / \mathrm{h}$ & $\mathcal{N}(120,0.3) \mathrm{km} / \mathrm{h}$ \\
\hline Max. acceleration & $1.5 \mathrm{~m} / \mathrm{s}^{2}$ & $2.9 \mathrm{~m} / \mathrm{s}^{2}$ \\
\hline Max. deceleration & $-3 \mathrm{~m} / \mathrm{s}^{2}$ & $-7.5 \mathrm{~m} / \mathrm{s}^{2}$ \\
\hline Car following model & $\mathrm{IDM}$ & Kraus
\end{tabular}

\section{A. Simulation Settings and Scenarios}

To assess the performance of the RATs, we vary the number of vehicles in the platoon (between 5 and 11), the IVD (between 5 and $15 \mathrm{~m}$ ), and the density of the surrounding traffic, as define in the previous section. After running a first set of experiments, we select the most challenging cases, that is for 11 platoon members driving with an IVD of $5 \mathrm{~m}$ and add vehicles in the vicinity of the highway. This yields more traffic density cases: $1500,3000,6000,9000$ and 12,000 vehicles/h in terms of total number of communicating vehicles in the simulation: The first two correspond to our low and medium volumes. Experiments are repeated 60 times.

\section{B. KPIs}

We analyze the performance of the RATs under the following communication and functional KPIs:

PER: ratio between the number of non-received packets and the total number of transmitted packets;

Delay: radio channel latency corresponding to signal processing delay on the physical layer, also including scheduling for LTE-V mode 3-it is averaged over all transmission occurring during a timestep;

IVD: bumper to hood distance between two vehicles when stopped after the emergency braking maneuver.

The two first KPIs are measured for the EM only, transmitted by the first vehicle several times after the hazard detection. Additionally, we count the total number of messages received without error.

\section{Simulation Results}

Our main communications KPIs are the PER and delay. We evaluate these two indicators whilst varying surrounding traffic (see Figs. 3 and 4). For IEEE 802.11p and LTE-V mode 4, the general trend is that the PER degrades when the surrounding traffic increases. For the former, increasing traffic also yields larger delays, though very limited. Moreover, the farther from the transmitter the poorer the PER. For the latter, the delays 


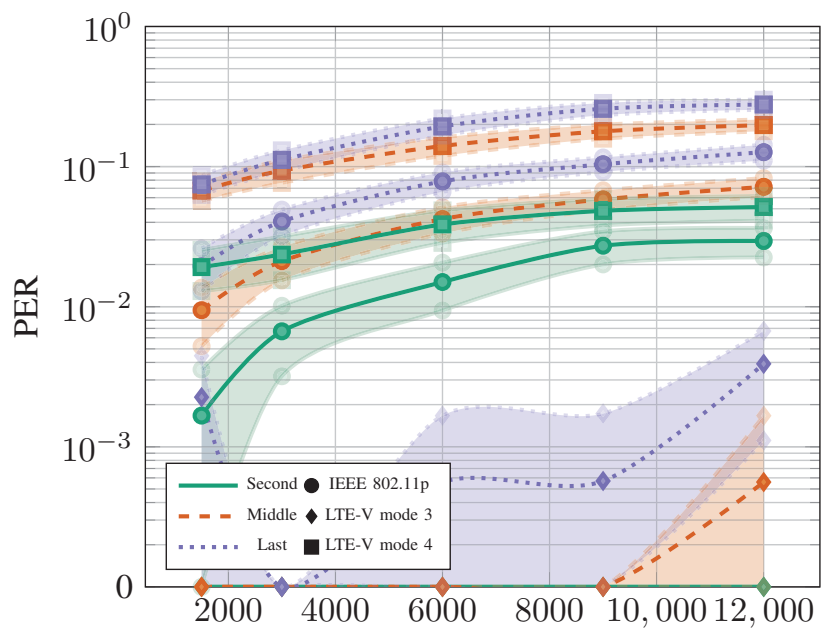

Surrounding traffic in vehicles $/ \mathrm{h}$

Fig. 3. Average PER affecting the link to the second (green plain), middle (orange dashed) and last (purple dotted) vehicles as a function of the traffic density. Results corresponding to IEEE 802.11p, LTE-V mode 3 and 4 are depicted with bullet, diamond and square markers respectively. The light areas correspond to the $95 \%$ confidence intervals.

are not constant but close to the same value, $12 \mathrm{~ms}$. However, the closer to the transmitter the larger the delays.

The PER of IEEE $802.11 \mathrm{p}$ is almost null with low surrounding traffic. This error rate reaches $10 \%$ at high surrounding density for the eleventh truck, which is at approximately $150 \mathrm{~m}$. In the meantime, the delay also increases but stays in very low range around $1 \mathrm{~ms}$.

For LTE-V mode 3 the PER is null or close to zero in every cases, with a slight dependency to the receiver distance and the surrounding traffic. This comes at a cost of a constant delay of $12 \mathrm{~ms}$. LTE-V mode 4 PER is rather low for the second vehicle, around $1 \%$, but reaches $10 \%$ for the last vehicle of the platoon.

Fig. 5 provides the number of successfully received messages for selected scenarios. In all scenarios, these numbers tend to only slightly vary when increasing the communication traffic, generally tending to decrease. However, the farther the receiver, the lower number of messages correctly received. The distance also accentuates the influence of the surrounding traffic. LTE-V mode 4 tends to have the lowest number of correctly received messages (less than $85 \%$ middle and last vehicles at large densities). For IEEE 802.11p, in every scenarios the received number of message is always very close to $100 \%$ correct, except for the farthest truck for which the former drops to $90 \%$. When using LTE-V mode 3, the number of correct messages received is constant around $100 \%$ for all trucks.

IVD is a functional KPI that provides insights on the impact of communications performances on the HDPL. Fig. 6 summarizes the results for varying surrounding traffic density. IVD, which illustrates the functional impact of communication at the vehicle level, stays rather close to the original distance, that is $5 \mathrm{~m}$, for the middle and last vehicles. It is between 3 and

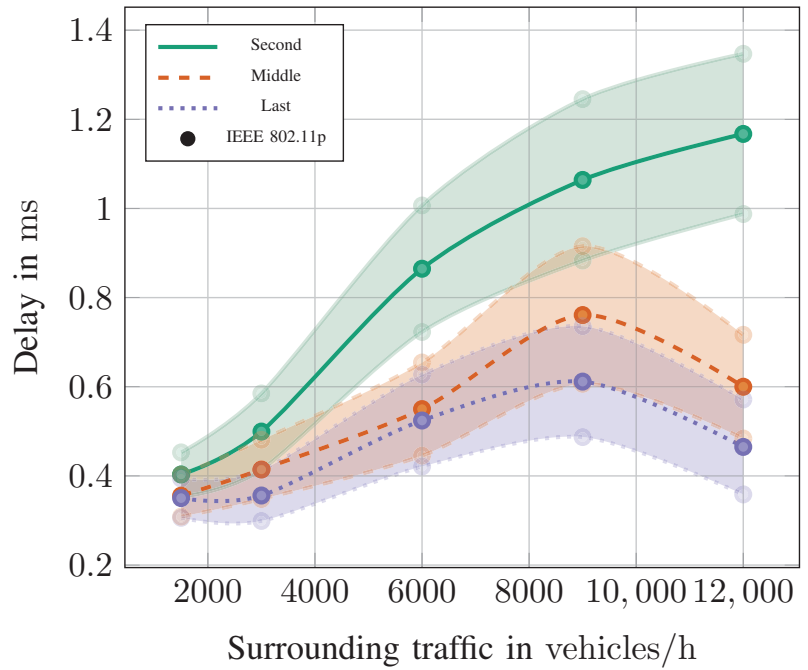

(a)

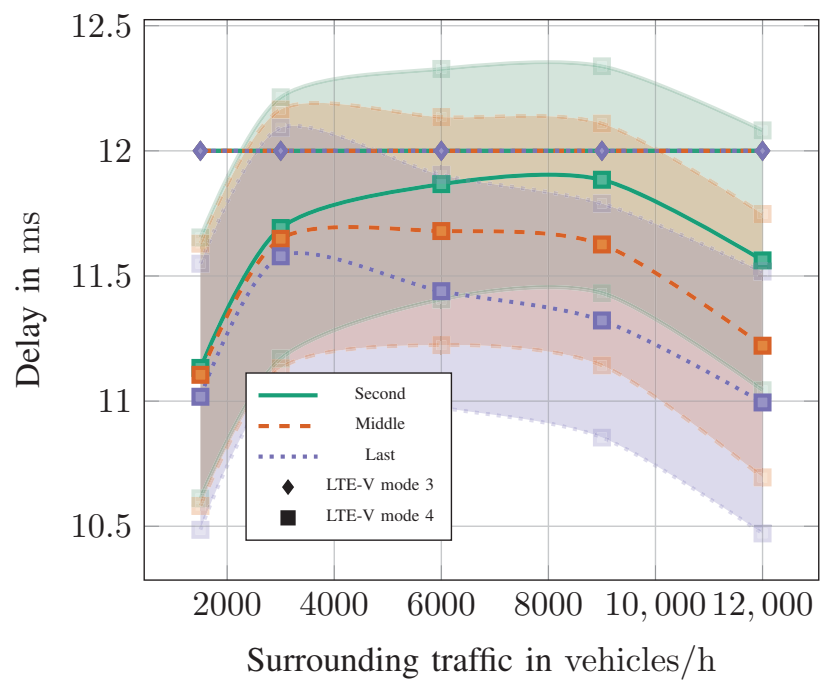

(b)

Fig. 4. Average delay affecting the link to the second (green plain), middle (orange dashed) and last (purple dotted) vehicles as a function of the traffic density. Results corresponding to (a) IEEE 802.11p, (b) LTE-V mode 3 and 4 are depicted with bullet, diamond and square markers respectively. The light areas correspond to the $95 \%$ confidence intervals.

$4 \mathrm{~m}$ when it comes to the second vehicle. With higher traffic density, some dangerous situations start to occur, especially for unmanaged technologies.

\section{Discussion}

In this section we derive insights on the impact of the environment on the system, from the channel to the application.

We first focus on the communications KPIs. Increasing the traffic density yields higher PER for both unmanaged technologies, IEEE 802.11p and LTE-V mode 4. The distance between the transmitter, here the first vehicle of the platoon, and the receiver has a similar effect. Both phenomena can be explained by higher level of interference affecting the communications. Subsequently, larger PER values result in lower probability to successfully receive a message. 


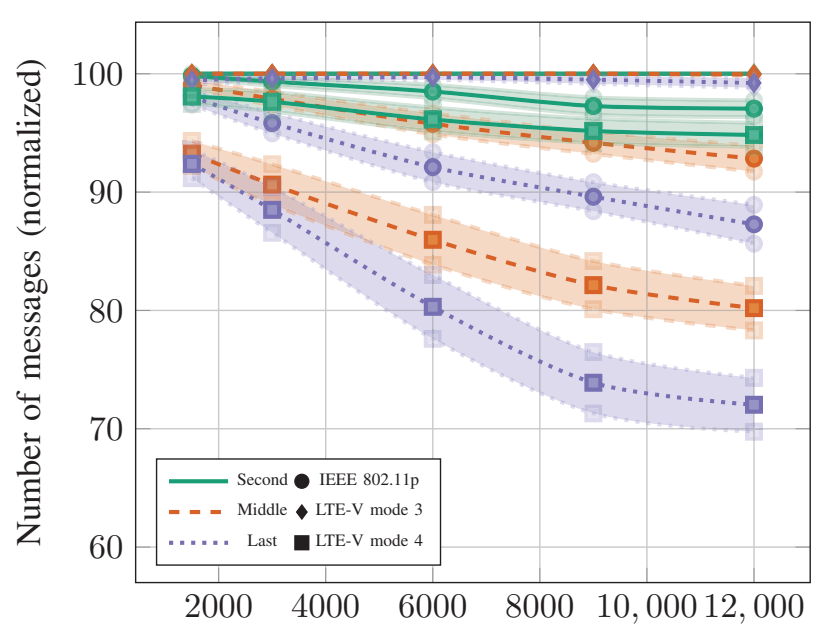

Surrounding traffic in vehicles $/ \mathrm{h}$

Fig. 5. Average number of messages received without error by the second (green plain), middle (orange dashed) and last (purple dotted) vehicles as a function of the traffic density. Results corresponding to IEEE 802.11p, LTE-V mode 3 and 4 are depicted with bullet, diamond and square markers respectively. The light areas correspond to the $95 \%$ confidence intervals.



Surrounding traffic in vehicles $/ \mathrm{h}$

Fig. 6. Average final IVD to the second (green plain), middle (orange dashed) and last (purple dotted) vehicles as a function of the traffic density. Results corresponding to IEEE $802.11 \mathrm{p}$, LTE-V mode 3 and 4 are depicted with bullet, diamond and square markers respectively. The light areas correspond to the 95\% confidence intervals.

Thanks to the successful resource allocation in the design of LTE-V mode 3, there is almost no packet error. Indeed, there are far less packet collisions with the scheduling management operated by eNBs, however resulting in a $12 \mathrm{~ms}$ constant scheduling delay. This fixed value is exceeded in very rare cases in which the cell is temporary overloaded, or when some lower priority but old messages are to be sent. Indeed, low priority messages reaching the timeout can usurp higher priority messages. It is worth noticing that the delays for low priority messages such as CAM is larger than $12 \mathrm{~ms}$ (not represented).
At the message level, the distance to the transmitter has the largest impact on the performance, especially for LTE-V mode 4. Common to all technologies, this trend is a direct consequence of the communication range, which is not a fixed value but dependent to the SINR (see Sec. II-C). While IEEE $802.11 \mathrm{p}$ and LTE-V mode 3 are almost not affected, at this level, by the increasing surrounding traffic, its variation accentuates the impact of the receiver distance on LTE-V mode 4 . The lower messages number for mode 4 gives an indication on the lower range.

Our EM is broadcast to all receivers in order to perform an emergency braking maneuver, which should be as synchronized as possible. Subsequently, the delays should be homogeneous in the platoon. This is however not the case in the experimental results. Furthermore, counter-intuitively, the closer the receiver, the larger the delay. This is intrinsic to the metric definition; indeed, it is an average delay over the received messages. Messages affected by high levels of interference are not received by farther receivers. These same messages are also likely to show higher delays. As a result, for far receivers, messages are received only in the best conditions, thus showing a lower average delay. This is also known as the survivorship bias. This explanation also holds for the larger confidence intervals at higher traffic densities.

In terms of variation to the growing surrounding traffic density, although slightly increasing, delays are rather constant for all RATs. In IEEE 802.11p, this increasing delay is caused by the higher message collision probability and the longer LBT time. Since our message is broadcast, no repetition mechanism intervenes and increases this delay. For the LTE-V RATs, the delay is $12 \mathrm{~ms}$ for mode 3 and stays in the $10.5 \mathrm{~ms}-12.5 \mathrm{~ms}$ range for mode 4 . The former delay, as previously mentioned, is the scheduling delay. The latter is a consequence of forcing subscribers to choose a SCI within $20 \mathrm{~ms}$ for this emergency message, yielding an average in this range.

Finally, as expected from the decreasing message reception probability with large traffic density, the risk of the emergency braking maneuver increases for the HDPL in such situations. Especially, the distance between the two first vehicles reaches below $3 \mathrm{~m}$ for medium to high traffic density, even below $2 \mathrm{~m}$ for high traffic density. Though $2 \mathrm{~m}$ is still a safe final IVD, this let draw the limits of the RATs for our application. The second truck is particularly affected by communications quality of service (QoS) variation as it is right behind the truck starting the maneuver. Indeed, in this instance, the first truck simultaneously brakes and sends the EM. Farther IVDs are very close to their original value $5 \mathrm{~m}$, meaning that they can tolerate higher PER. This holds already for the second IVD, the final distance between the second and third trucks (not represented). Considering that links to farther receivers are affected by higher PER, this is promising for the scalability of the platooning concept.

\section{CONCLUSION}

In this report, we have presented the implementation and performance analysis of a HDPL emergency braking maneu- 
ver supported by communications using three RATs: IEEE 802.11p, LTE-V mode 3 and mode 4. In order to compare the performance of the RATs, we consider different surrounding traffic densities. To also take the distance between the transmitter and the receiver into account, we consider three representative vehicles: the second, the middle and the last one. We assess the performance in terms of PER, delay, number of received messages and IVD.

For IEEE 802.11p and LTE-V mode 4, our two unmanaged RATs, PER increases with growing surrounding traffic density. This indicator also increases when increasing the distance to the receiver. For the LTE-V mode 3, this two variables have little influence on the PER, thanks to scheduling. The delay when using IEEE 802.11p increases in a similar way as the observed PER with increasing traffic density.

Though the emergency maneuver is generally performed in the safe manner, we observe that we are reaching the safety boundaries for high traffic density (12,000 vehicles/h), especially for LTE-V mode 4 and for the second truck. If the PER and delay are known in advance, and the distance to the hazard allows to slightly delay the emergency braking, it could be envisioned that the platoon braking maneuver accounts for this expected QoS. This motivates the prediction of QoS (PQoS) concept, a promising development of recent communications systems.

In a further analysis, we intend to use this benchmark framework with ray tracing instead of the stochastic channel models and to investigate new $5 \mathrm{G}$ components.

\section{REFERENCES}

[1] C. Sommer, R. German, and F. Dressler, "Bidirectionally coupled network and road traffic simulation for improved IVC analysis," IEEE Transactions on Mobile Computing, vol. 10, no. 1, pp. 3-15, January 2011.

[2] A. Varga and R. Hornig, "An overview of the OMNeT++ simulation environment," in Proceedings of the 1st international conference on Simulation tools and techniques for communications, networks and systems \& workshops. ICST (Institute for Computer Sciences, SocialInformatics and Telecommunications Engineering), 2008, p. 60.

[3] D. Krajzewicz, J. Erdmann, M. Behrisch, and L. Bieker, "Recent development and applications of SUMO - Simulation of Urban MObility," International Journal On Advances in Systems and Measurements, vol. 5, no. 3\&4, pp. 128-138, December 2012.

[4] F. Hagenauer, F. Dressler, and C. Sommer, "A simulator for heterogeneous vehicular networks," in 6th IEEE Vehicular Networking Conference (VNC 2014), Poster Session. Paderborn, Germany: IEEE, December 2014, pp. 185-186.
[5] R. Riebl, H.-J. Günther, C. Facchi, and L. Wolf, "Artery - Extending Veins for VANET applications," in 4th International Conference on Models and Technologies for Intelligent Transportation Systems (MTITS 2015). Budapest, Hungary: IEEE, Jun 2015.

[6] M. Segata, S. Joerer, B. Bloessl, C. Sommer, F. Dressler, and R. Lo Cigno, "PLEXE: A platooning extension for veins," in 6th IEEE Vehicular Networking Conference (VNC 2014). Paderborn, Germany: IEEE, December 2014, pp. 53-60.

[7] A. Virdis, G. Stea, and G. Nardini, "Simulating LTE/LTE-Advanced networks with SimuLTE," in Simulation and Modeling Methodologies, Technologies and Applications. Springer, 2015, pp. 83-105.

[8] G. Nardini, A. Virdis, and G. Stea, "Simulating device-to-device communications in OMNeT++ with SimuLTE: scenarios and configurations," arXiv preprint arXiv:1609.05173, 2016.

[9] G. F. Riley and T. R. Henderson, "The ns-3 network simulator," in Modeling and tools for network simulation. Springer, 2010, pp. 15-34.

[10] Y. Pigné, G. Danoy, and P. Bouvry, "A platform for realistic online vehicular network management," in GLOBECOM Workshops (GC Wkshps), 2010 IEEE. IEEE, 2010, pp. 595-599.

[11] R. Rouil, F. Cintrón, A. B. Mosbah, and S. G. Quintiliani, “A Long Term Evolution (LTE) device-to-device module for ns-3," in The Workshop on $n s-3$ (WNS3), 2016.

[12] O. Michel, "Cyberbotics Ltd. Webots" ${ }^{\mathrm{TM}}$ : professional mobile robot simulation," International Journal of Advanced Robotic Systems, vol. 1, no. 1, p. $5,2004$.

[13] I. Llatser, G. Jornod, A. Festag, D. Mansolino, I. Navarro, and A. Martinoli, "Simulation of cooperative automated driving by bidirectional coupling of vehicle and network simulators," in Intelligent Vehicles Symposium (IV), 2017 IEEE. IEEE, 2017, pp. 1881-1886.

[14] T. Jansen, Optimisation of the Handover Decision in Infrastructure Networks Using Realistic Simulation Environments. Shaker Verlag $\mathrm{GmbH}$, Germany, 2016.

[15] "Study on LTE device to device proximity services - Radio aspects," 3GPP, Tech. Rep. 36.843 v12.1.0, Mar. 2014.

[16] "Intelligent Transport Systems (ITS); Performance evaluation of selforganizing TDMA as medium access control method applied to ITS; Access layer part," European Telecommunications Standards Institute, Tech. Rep. ETSI TR 102862 V1.1.1, 2011.

[17] "Intelligent Transport Systems (ITS); Access layer specification for intelligent transport systems operating in the $5 \mathrm{GHz}$ frequency band," European Telecommunications Standards Institute, Tech. Rep. ETSI EN 302663 V1.2.0, 2012.

[18] "Vehicle to Vehicle (V2V) services based on LTE sidelink; User Equipment (UE) radio transmission and reception," 3GPP, Tech. Rep. 36.785 v14.0.0, Sep. 2016.

[19] "Evolved Universal Terrestrial Radio Access (E - UTRA); Physical layer procedures ," 3GPP, Tech. Rep. 36.213 v14.3.0, Jun. 2017.

[20] "Evolved Universal Terrestrial Radio Access (E - UTRA); Medium Access Control (MAC)," 3GPP, Tech. Rep. 36.321 v14.3.0, Jun. 2017.

[21] V. Shivaldova, G. Maier, D. Smely, N. Czink, A. Paier, and C. Mecklenbräuker, "Performance analysis of vehicle-to-vehicle tunnel measurements at $5.9 \mathrm{GHz}$," in General Assembly and Scientific Symposium, 2011 URSI. IEEE, 2011, pp. 1-4

[22] S. Hahn, "Mobile radio network management in the context of realistic heterogeneous scenarios," Ph.D. dissertation, Technische Universität Braunschweig, 2017, sec. 2.3.1. 